H. Umemura

Nagoya Math. J.

Vol. 59 (1975), 107-134

\title{
STABLE VECTOR BUNDLES WITH NUMERICALLY TRIVIAL CHERN CLASSES OVER A HYPERELLIPTIC SURFACE
}

\author{
HIROSHI UMEMURA
}

In [17], Weil studied the space of representations of certain Fuchsian groups as a generalization of Jacobian variety. The theory of stable vector bundles over a curve developed by Mumford, Seshadri and others are the theory of unitary representations of Fuchsian groups. The moduli space of stable vector bundles over a curve is the space of the irreducible unitary representations of a Fuchsian group. The moduli space is studied in detail. Recently Mumford (unpubished) and Takemoto [12] introduced the notion of $H$-stable vector bundle over a non-singular projective algebraic surface. In this paper, we study the space of the irreducible unitary representations of the fundamental group of a hyperelliptic surface. Our view point is based on the theory of $H$-stable vector bundles of Takemoto [12] and [13]. We deal only with hyperelliptic surfaces. Our results should be generalized to the vector bundles over some other surfaces (See $\S 3$ ). Our main results are as follows:

$1^{\circ}$ An $H$-stable vector bundle with numerically trivial Chern classes over a hyperelliptic surface is defined by an irreducible unitary representation of the fundamental group.

$2^{\circ}$ The space of irreducible unitary representations gives the local moduli if degree $\geq 2$ with some additional assumption (see Theorem (2.18) for a precise statement).

$3^{\circ}$ There are many $H$-semi-stable vector bundles which can not be a limit of $H$-stable bundles. In certain case, it is impossible to deform an $H$-stable bundle of certain rank $r$ with numerically trivial Chern classes to any $H$-semi-stable vector bundle which is not $H$-stable.

Our theory can be regarded as a generalization of the Picard scheme. $2^{\circ}$ shows the space of irreducible unitary representation of the fundamental group sometimes gives finer result than the Picard scheme.

Received August 30, 1974. 


\section{§ 1. $H$-stable vector bundles}

We recall the definition and some basic results on $H$-stable vector bundles (for the proof see Takemoto [12]). We assume that all schemes, which we call varieties, are reduced and of finite type over the complex number field $\boldsymbol{C}$.

DEFINITION (1.1). Let $S$ be a non-singular projective surface. Let $H$ be an ample line bundle on $S$. Let $E$ be a vector bundle i.e. a locally free sheaf of finite rank on $S$. We say that $E$ is $H$-stable (resp. $H$-stable) if we have

$$
\begin{gathered}
\frac{\left(H \cdot c_{1}(E)\right)}{r(E)}<\frac{\left(H \cdot c_{1}(F)\right)}{r(F)} \\
(\text { resp. } \leq)
\end{gathered}
$$

for any torsion free quotient $O_{S}$-Module $F$ of $E$ with $r(E)>r(F)$ where $c_{1}()$ is the first Chern class, $r()$ is the rank and $(\cdot)$ denotes the intersection number.

If we fix the line bundle $H$ and the numerical Chern classes $c_{1}, c_{2}$ and if $r=2$, the coarse moduli space of $H$-stable vector bundles $E$ with $c_{1}(E)=c_{1}, c_{2}(E)=c_{2}$, exists (Maruyama [4], see also Theorem (2.16)).

Let $\tilde{S}$ be the universal covering space of $S$. Let $G$ be the fundamental group of $S$ hence $G$ acts on $\tilde{S}$ and the quotient space $\tilde{S} / G$ is isomorphic to $S$. Given a representation $\rho: G \rightarrow G L(r, C)$, we can associate a vector bundle $E$ to this representation.

Proposition (1.2). If $\rho$ is a unitary representation, $E_{\rho}$ is $H$-semistable for any ample line bundle $H$ on $S$. If $\rho$ is an irreducible unitary representation, $E_{\rho}$ is $H$-stable for any ample line bundle $H$ on $S$.

Proof (essentially due to $\mathrm{F}$. Takemoto). Let $H$ be an ample line bundle on $S$. Assume that $\rho$ is an irreducible unitary representation. We show that $E_{\rho}$ is $H$-stable. We remark that a vector bundle is $H$ stable if and only if it is $H^{\otimes m}$-stable for one positive integer $m$. Hence we may replace $H$ by $H^{\otimes m}$. Let $C_{m}$ be a generic hyperplane section of the linear system $\left|H^{\otimes m}\right|$. Then, taking $m$ sufficiently big, we have, by the Lefschetz theorem, the map $\pi_{1}\left(C_{m}\right) \stackrel{i_{*}}{\longrightarrow} \pi_{1}(S)$ is surjective (Bott [2]). Hence the unitary representation $\rho \circ i_{*}$ is irreducible. The restriction 
$E_{\rho \mid C_{m}}$ of $E_{\rho}$ to $C_{m}$ is associated to the irreducible unitary representation hence by Narasimhan and Seshadri [8] $E_{\rho \mid C_{m}}$ is stable. It follows that $E_{\rho}$ is $H^{\otimes m}$-stable. This is what we had to show. When $\rho$ is a unitary representation, the proof of $H$-semistability of $E_{\rho}$ is the same as above, in fact easier.

Proposition (1.3). Let $S$ be a non-singular projective surface. Let $T$ be a variety. Let $E$ be vector bundle over $T \times S$ such that for any (closed) point $t$, the restriction $E_{t}$ of $E$ to $t \times S$ has numerically trivial Chern classes and is $H_{t}$-stable for some ample line bundle $H_{t}$ on $S$. Then the set of points $\left\{t \in T \mid E_{t}\right.$ is defined by an irreducible unitary representation\} is closed.

Proof. Let $G$ be the fundamental group of $S$. Let $g_{1}, g_{2}, \cdots, g_{\ell}$ be a set of generators of $G$. Let $\rho$ be a representation of $G$. Then it determines a point $\left(\rho\left(g_{1}\right), \rho\left(g_{2}\right), \cdots, \rho\left(g_{\ell}\right)\right) \in G L(r, C)^{\ell}$. It follows easily all the representations of degree $r$ of $G$ (not the isomorphism classes) form a closed analytic subset $W \subset G L(r, C)^{\ell}$. The intersection $U=$ $W \cap U(r, C)^{\ell}$ is a compact subset of $W$. This is the set of all unitary representation of $G$. Let $F$ be the family of vector bundles over $S \times W$ i.e. the restriction $F_{w}$ of $F$ to $S \times w$ is the vector bundle associated to the representation corresponding to $w$. Consider the set $M=\{(w, k) \in W$ $\left.\times T \mid \operatorname{Hom}\left(F_{w}, E_{k}\right) \neq 0\right\}$. Then $M$ is a closed subset of $W \times T$. If we consider the projection $\operatorname{pr}_{T}(M \cap U \times T)$, then this is a closed subset of $T$ since $U$ is compact. Proposition now follows if we notice the following fact; Let $E_{1}$ be an $H$-semi-stable bundle and $E_{2}$ an $H$-stable bundle such that $E_{1}$ and $E_{2}$ have the same numerical Chern classes and $r\left(E_{1}\right)=$ $r\left(E_{2}\right)$. If $\operatorname{Hom}\left(E_{1}, E_{2}\right) \neq 0$, then $E_{1}$ is isomorphic to $E_{2}$.

Proposition (1.4). Let $S$ be a compact complex manifold. Let $\rho_{1}$ and $\rho_{2}$ be unitary representations of the fundamental group of $S$. We denote by $E_{\rho_{1}}, E_{\rho_{2}}$ the vector bundles associated to the unitary representations $\rho_{1}$ and $\rho_{2}$. Then the vector bundle $E_{\rho_{1}}$ is isomorphic to $E_{\rho_{2}}$ if and only if the representations $\rho_{1}$ and $\rho_{2}$ are equivalent.

Proof. See Seshadri [10]. 


\section{$\S 2$. Moduli space of $H$-stable vector bundles with trivial Chern classes over hyperelliptic surfaces}

We need some results on hyperelliptic surfaces. For the detail we refer to Suwa [11].

(2.1.1) A hyperelliptic surface $S$ is, by definition, an elliptic surface free from singular fibres over an elliptic curve with the first Betti number $b_{1}(S)=2$.

(2.1.2) Let $K$ be the canonical bundle of a hyperelliptic surface $S$. Then according to Suwa [11], such surfaces are classified into four types:

I) $K^{\otimes 2} \simeq O_{s} \quad\left(K \neq O_{s}\right)$

II) $K^{\otimes 3} \simeq O_{s} \quad\left(K \neq O_{s}\right)$

III) $K^{\otimes 4} \simeq O_{s} \quad\left(K^{\otimes 2} \neq O_{t}\right)$

IV) $K^{\otimes 6} \simeq O_{s} \quad\left(K^{\otimes 2}, K^{\otimes_{3}} \neq O_{s}\right)$.

The hyperelliptic surface has an abelian variety as an unramified covering space of degree 2,3,4 or 6 according as $S$ is of type I, II, III or IV. Let $d(S)$ be the minimal positive integer such that $K^{\otimes d(S)}$ is isomorphic to $O_{S}$.

We quote

THEOREM (2.1.3) (Suwa [11] p. 473). Any hyperelliptic surface can be expressed as the quotient space of an abelian variety $A$ by the group generated by an automorphism $g_{5}$ of $A$. The period matrix of $A$ and the automorphism $g_{5}$ are given as follows

I)

i) $\left(\begin{array}{llll}1 & 0 & \tau & 0 \\ 0 & 1 & 0 & \omega\end{array}\right), \quad$ ii) $\left(\begin{array}{cccc}1 & 0 & \tau & 0 \\ 0 & 1 & \frac{1}{2} & \omega\end{array}\right)$,

$$
g_{5}:(u, \zeta) \longmapsto\left(u+\frac{1}{2},-\zeta\right) \text {, }
$$

II) ii) $\left(\begin{array}{llll}1 & 0 & \tau & 0 \\ 0 & 1 & 0 & \rho^{2}\end{array}\right), \quad$ ii) $\left(\begin{array}{cccc}1 & 0 & \tau & 0 \\ 0 & 1 & \frac{1}{3}\left(1-\rho^{2}\right) & \rho^{2}\end{array}\right)$,

$$
g_{5}:(u, \zeta) \longmapsto\left(u+\frac{1}{3}, \rho^{2} \zeta\right), \quad \rho=\exp (\pi i / 3),
$$

III) iii) $\left(\begin{array}{llll}1 & 0 & \tau & 0 \\ 0 & 1 & 0 & i\end{array}\right), \quad$ iii) $\left(\begin{array}{cccc}1 & 0 & \tau & 0 \\ 0 & 1 & \frac{1}{2}(1+i) & i\end{array}\right)$,

$$
g_{5}:(u, \zeta) \longmapsto\left(u+\frac{1}{4}, i \zeta\right),
$$

IV)

$$
\left(\begin{array}{cccc}
1 & 0 & \tau & 0 \\
0 & 1 & 0 & \rho^{2}
\end{array}\right), \quad g_{5}:(u, \zeta) \longmapsto\left(u+\frac{1}{6},-\rho^{2} \zeta\right),
$$


where $\tau$ and $\omega$ denote arbitrary constants with non-zero imaginary parts.

The parameters $\tau, \omega$ in the case $I$ and $\tau$ in the other cases give the local moduli of hyperelliptic surfaces. They form complex analytic families of hyperelliptic surfaces and the parameters $\tau, \omega$ and $\tau$ are effective and complete.

In any case of seven types above, we denote by $g_{i}, 1 \leq i \leq 4$, the $i$-th column vector of the period matrix. Then $\left\{g_{1}, g_{2}, g_{3}, g_{4}, g_{5}\right\}$ is a set of generators of the fundamental group.

(2.1.4) If we put $G=\pi_{1}(S)$ and $H=$ the normal subgroup of $G$ generated by $g_{1}, g_{2}, g_{3}$ and $g_{4}$, then $H$ is isomorphic to $Z^{\otimes^{4}}$ and we get an exact sequence

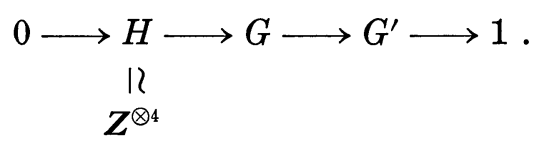

The quotient group $G^{\prime}$ is generated by $g_{5} \bmod H$ and of order $2,3,4,6$ according as $S$ is of type I, II, III, IV.

LEMMA (2.1.5). Suppose that $S$ is of type I i), then we have following relations

(0) $g_{1}, g_{2}, g_{3}$ and $g_{4}$ commute each other.

(a) $g_{1} g_{5}=g_{5} g_{1}$

(b) $g_{2} g_{5}=g_{5} g_{2}^{-1}$

(c) $g_{3} g_{5}=g_{5} g_{3}$

(d) $g_{4} g_{5}=g_{5} g_{4}^{-1}$

(e) $g_{5}^{2}=g_{1}$.

Any other relation is deduced from the above relations.

Proof. It is trivial that $g_{1}, g_{2}, g_{3}, g_{4}$ and $g_{5}$ satisfy the condition (0), (a), (b), (c), (d) and (e). Conversely consider the group $\Gamma$ generated $\gamma_{1}, \gamma_{2}$, $\gamma_{3}, \gamma_{4}$ and $\gamma_{5}$ such that $\gamma_{1}, \gamma_{2}, \gamma_{3}, \gamma_{4}$ and $\gamma_{5}$ satisfy the relations above. Let $N^{\prime}$ be a subgroup of $\Gamma$ generated by $\gamma_{1}, \gamma_{2}, \gamma_{3}, \gamma_{4}$. $N^{\prime}$ is a normal subgroup. We have

$$
0 \longrightarrow N^{\prime} \longrightarrow \Gamma \longrightarrow \Gamma / N^{\prime} \longrightarrow 1
$$

But the relations above also determine the cocycle of the extention above. For the same reason we have following lemmae.

LEMMA (2.1.6). If $S$ is of type I ii), then we have following relations 
(0) $g_{1}, g_{2}, g_{3}$ and $g_{4}$ commute each other.
(a) $g_{1} g_{5}=g_{5} g_{1}$
(b) $g_{2} g_{5}=g_{5} g_{2}^{-1}$
(c) $g_{3} g_{5}=g_{5} g_{3} g_{2}^{-1}$
(c) $g_{4} g_{5}=g_{5} g_{4}^{-1}$
(e) $g_{5}^{2}=g_{1}$

Any other relation is deduced from the above relations.

LEMMA (2.1.7). If $S$ is of type II $i$ ), then we have following relations

(0) $g_{1}, g_{2}, g_{3}$ and $g_{4}$ commute each other.
(a) $g_{1} g_{5}=g_{5} g_{1}$
(b) $g_{2} g_{5}=g_{5} g_{2}^{-1} g_{4}^{-1}$
(c) $g_{3} g_{5}=g_{5} g_{3}$
(d) $g_{4} g_{5}=g_{5} g_{2}$
(e) $g_{5}^{3}=g_{1}$

Any other relation is deduced from the above relations.

LEMMA (2.1.8). If $S$ is of type II ii), then we have following relations

(0) $g_{1}, g_{2}, g_{3}$ and $g_{4}$ commute each other.
(a) $g_{1} g_{5}=g_{5} g_{1}$
(b) $g_{2} g_{5}=g_{5} g_{2}^{-1} g_{4}^{-1}$
(c) $g_{3} g_{5}=g_{5} g_{3} g_{2}^{-1}$
(d) $g_{4} g_{5}=g_{5} g_{2}$
(e) $g_{5}^{3}=g_{1}$

Any other relation is deduced from the above relations.

LEMMA (2.1.9). If $S$ is of type III i), then we have following relations (0) $g_{1}, g_{2}, g_{3}$ and $g_{4}$ commute each other.
(a) $g_{1} g_{5}=g_{5} g_{1}$
(b) $g_{2} g_{5}=g_{5} g_{4}^{-1}$
(c) $g_{3} g_{5}=g_{5} g_{3}$
(d) $g_{4} g_{5}=g_{5} g_{2}$
(e) $g_{b}^{4}=g_{1}$

Any other relation is deduced from the above relations.

LEMMA (2.1.10). If $S$ is of type III ii), then we have following relations

(0) $g_{1}, g_{2}, g_{3}$ and $g_{4}$ commute each other.
(a) $g_{1} g_{5}=g_{5} g_{1}$
(b) $g_{2} g_{5}=g_{5} g_{4}^{-1}$
(c) $g_{3} g_{5}=g_{5} g_{3} g_{4}^{-1}$
(d) $g_{4} g_{5}=g_{5} g_{2}$
(e) $g_{5}^{4}=g_{1}$

Any other relation is deduced from the above relations. 
LEMMA (2.1.11). If $S$ is of type IV, then we have following relations

(0) $g_{1}, g_{2}, g_{3}$ and $g_{4}$ commute each other.

$\begin{array}{ll}\text { (a) } g_{1} g_{5}=g_{5} g_{1} & \text { (b) } g_{2} g_{5}=g_{5} g_{2} g_{4}\end{array}$

$\begin{array}{ll}\text { (c) } g_{3} g_{5}=g_{5} g_{3} & \text { (d) } g_{4} g_{5}=g_{5} g_{2}^{-1}\end{array}$

(e) $g_{5}^{6}=g_{1}$.

Any other relation is deduced from the above relations.

LemMa (2.2) (Oda). Let $A$ be an abelian variety. Let $E$ be a vector bundle of rank $r$ over $A$ and $p: H^{1}\left(A, \Omega_{A}^{1}\right) \rightarrow H^{1}\left(A, \Omega_{A}^{1} \otimes\right.$ End $\left.E\right)$ be the linear map induced by the canonical inclusion $0 \rightarrow O_{A} \rightarrow$ End $E$. Let $a(E) \in H^{1}\left(A, \Omega_{A}^{1} \otimes\right.$ End $\left.E\right)$ be the fundamental class of $E$. If $a(E)$ is in $(1 / r) p\left(H^{2}(A, Z) \cap H^{1}\left(A, \Omega_{A}^{1}\right)\right)$, then there exists a line bundle $L$ on $A$ such that $L^{-1} \otimes r_{A}^{*} E$ is homogeneous, where $r_{A}$ is the multiplication by $r$.

Proof. Let $\pi: C^{n} \rightarrow A$ be the universal covering space of $A$. Let $\Gamma$ be the fundamental group of $A$. Hence $C^{n} / \Gamma \simeq A$. Since $H^{i}\left(C^{n}, \pi^{*} \Omega_{A}^{1}\right)$ $=0 i \geq 1$, we have

$$
H^{i}\left(\Gamma, H^{0}\left(C^{n}, \pi^{*} \Omega_{A}^{1}\right)\right) \simeq H^{i}\left(A, \Omega_{A}^{1}\right)
$$

where the left hand side is the $i$-th cohomology group of $\Gamma$-module $H^{0}\left(C^{n}, \pi^{*} \Omega_{A}^{1}\right)$ (see Mumford [7]). For the same reason,

$$
H^{i}\left(\Gamma, H^{0}\left(C^{n}, \pi^{*}\left(\Omega_{A}^{1} \otimes \text { End } E\right)\right)\right) \simeq H^{i}\left(A, \Omega_{A}^{1} \otimes \text { End } E\right) .
$$

Let $\rho_{u}(z)$ be a 1-cocycle for $\Gamma$ with coefficients in $H^{0}\left(C^{n}, G L\left(r, O_{C^{n}}\right)\right)$ defining $E$. Then $a(E)$ corresponds to the cocycle $-d \log \rho_{u}(z)=-d \rho_{u}(z) \cdot \rho_{u}(z)^{-1}$. Hence from the hypothesis and Weil [18], there exists a 1-cocycle $\varphi_{\alpha}(z)$ for $\Gamma$ with coefficients in $H^{0}\left(C^{n}, O_{C^{n}}^{*}\right)$ such that $-d \log \rho_{u}(z)=-(1 / r) d \log \varphi_{\alpha}(z) \cdot I_{r}$ in $H^{1}\left(\Gamma, H^{0}\left(C^{n}, \pi^{*}\left(\Omega_{A}^{1} \otimes\right.\right.\right.$ End $\left.\left.E\right)\right)$. It is easy to see that $r_{A}^{*}\left(-(1 / r) d \log \varphi_{\alpha}(z)\right)$ is integral i.e. $-(1 / r) d \log \varphi_{\alpha}(z) \in H^{1}\left(C^{n}, \pi^{*} \Omega_{A^{\prime}}^{1}\right)$ corresponds to an element of the subset $H^{2}\left(A^{\prime}, Z\right) \cap H^{1}\left(A^{\prime}, \Omega_{A^{\prime}}^{1}\right)$ of $H^{1}\left(A^{\prime}, \Omega_{A^{\prime}}^{1}\right)$ where $\Gamma^{\prime} \subset r \Gamma$ and $A^{\prime}$ $=C^{n} / \Gamma^{\prime}$. By Weil [18], there exists a line bundle $L$ on $A^{\prime}$ defined by a 1-cocycle $\varphi_{u}^{\prime}$ for $\Gamma^{\prime}$ with coefficients in $H^{0}\left(C^{n}, O_{\boldsymbol{C}^{n}}^{*}\right)$ such that $d \log \varphi_{u}^{\prime}(z)=$ $(1 / r) d \log \varphi_{u}(z), u \in \Gamma^{\prime}$. Hence $-d \log \rho_{u}(z)=-d \log \varphi_{u}^{\prime}(z) I_{r}, u \in \Gamma^{\prime}$. If we consider the vector bundle $L^{-1} \otimes \varphi^{*} E$ on $A^{\prime}$, this vector bundle is defined by the 1-cocycle $\varphi_{u}^{\prime}(z)^{-1} \rho_{u}(z)$ for $\Gamma$ with coefficients in $H^{0}\left(C^{n}, G L\left(r, O_{\boldsymbol{C}^{n}}\right)\right)$. Then $-d \log \varphi_{u}^{\prime}(z) \rho_{u}(z)=0$ in $H^{1}\left(A^{\prime}, \pi^{\prime *}\left(\Omega_{A^{\prime}}^{1} \otimes \operatorname{End}\left(L^{-1} \otimes E\right)\right)\right.$. Hence 
$L^{-1} \otimes \varphi^{*} E$ has a connection (see Atiyah [1]). By the theorem of Matsushima [5], $L^{-1} \otimes \varphi^{*} E$ is homogeneous.

CoRollary (2.3). Let $A$ be an abelian variety of dimension 2 and $E$ an $H$-stable vector bundle. If $c_{1}=0, c_{2}=0$, then $E$ is a line bundle.

Proof. Since an $H$-stable vector bundle i.e. $\operatorname{dim} H^{0}(A$, End $E)=1$, by the Riemann-Roch theorem, the canonical linear map $H^{1}\left(A, \Omega_{A}^{1}\right) \rightarrow$ $H^{1}\left(A, \Omega_{A}^{1} \otimes\right.$ End $\left.E\right)$ is an isomorphism. Hence there exist an isogeny $\varphi: A^{\prime} \rightarrow A$ and a line bundle $L$ such that $L^{-1} \otimes \varphi^{*} E$ is homogeneous. Since the first Chern class of $E$ is trivial, we can take $L \simeq O_{A^{\prime}}$. On the other hand, by Takemoto [13], $\varphi^{*} E$ is the direct sum of $\varphi^{*} H$-stable bundles. By Morimoto [6], $\varphi^{*} E$ is the direct sum of line bundles which are algebraically equivalent to 0 . Hence $E$ is the direct image of a line bundle on $A^{\prime}$. Therefore the rank should be one.

Corollary (2.4) (Oda). Let $A$ be an abelian variety of Dimension 2. Let $E$ be an $H$-stable vector bundle of rank $r$ with $(r-1) c_{1}^{2}-2 r c_{2}$ $=0$. Then there there exist an isogeny $\varphi: A^{\prime} \rightarrow A$ and a line bundle $L$ on $A^{\prime}$ such that $\varphi_{*} L \simeq E$.

Proof. Similar to the proof of Corollary (2.3).

Lemma (2.5). Let $A$ be an abelian variety of Dimension 2. Let $E$ be a vector bundle on $A$. Then,

(1) The second Chern class of End $E=-(r-1) c_{1}^{2}+2 r c_{2}$ where $c_{1}, c_{2}$ denote the first and the second Chern class of $E$.

(2) If $E$ is simple, then $(r-1) c_{1}^{2}-2 r c_{2} \leq 0$.

Proof. (2) follows from the Riemann-Roch theorem for End $E$. (1) is trivial.

LEMMA (2.6). Using the notation of Theorem (2.1.3), let $E$ be an $H$-stable vector bundle of rank $r$ over the hyperelliptic surface $S$. If $E$ has the numerically trivial Chern classes, then $\pi^{*} E$ is the direct sum of line bundles which are algebraically equivalent to 0 , where $\pi: A \rightarrow S$ is the natural projection.

Proof. We prove the lemma when $S$ is of type I. The proofs for 
other cases are similar. By Takemoto [13], $\pi^{*} E$ is either $\pi^{*} H$-stable or isomorphic to $E_{1} \oplus \bar{g}_{5}^{*} E_{1}$ where $E_{1}$ is a $\pi^{*} H$-stable over $A$. If $\pi^{*} E$ is $\pi^{*} H$-stable, then $\pi^{*} E$ is line bundle by Lemma (2.3). Hence we may assume $\pi^{*} E \simeq E_{1} \oplus \bar{g}_{5}^{*} E_{1}$. We claim that $E_{1}$ has numerically trivial Chern classes. We put $r_{1}=\operatorname{rank} E_{1}$. Let $g: C^{2} \rightarrow C^{2}$ be a linear automorphism of the universal

$$
(u, \zeta) \longmapsto(u, \zeta)
$$

covering space of $A$. We denote by $\bar{g}$ the automorphism of $A$ induced by $g$. Putting $E_{2}=\bar{g}_{5}^{*} E_{1}$ let $c^{2}$ be the $i$-th Chern class of $E_{1}, 1 \leq i \leq 2$. Then, from the hypothesis $E \simeq E_{1} \oplus \bar{g}_{5}^{*} E_{1}$, we get

$$
c_{1}+\bar{g}^{*} c_{1}=0, \quad\left(c_{1} \cdot \bar{g}^{*} c_{1}\right)+2 c_{2}=0,
$$

since a translation of a line bundle is algebraically equivalent to itself. It follows $-\left(c_{1}^{2}\right)+2 c_{2}=0$. Let $E_{1}=L(\bar{H}, \alpha)$ (see Mumford [7], p. 20). Then it also follows $\bar{H}+\left(\begin{array}{rr}1 & 0 \\ 0 & -1\end{array}\right) \bar{H}\left(\begin{array}{rr}1 & 0 \\ 0 & -1\end{array}\right)=0$. Hence $\bar{H}=\left(\begin{array}{ll}0 & a \\ \bar{a} & 0\end{array}\right)$. In view of Lemma (2.3), it is sufficient to show $a=0$. If $a \neq 0$, the index of $L$ would be 1 since $\bar{H}$ has 1 positive eigen-value and 1 negative one (see Mumford [8] and Umemura [15]). Hence by the Riemann-Roch theorem, $c_{1}^{2}<0$. Hence $c_{2}>0$ since $-\left(c_{1}^{2}\right)+2 c_{2}=0 . \quad(r-1) c_{1}^{2}-2 r c_{2}=$ $(r-1)\left(c_{1}^{2}-2 c_{2}\right)+2 c_{2}=2 c_{2}>0$ contradicting to Lemma (2.5). Hence a should be 0 .

CoRollary (2.7). Using the notation of Lemma (2.6), we assume that $E$ has numerically trivial Chern classes. Then $r \mid d(S)$ and there exist an unramified covering of degree $d(s) \mid r$ of $S$ and a line bundle on it such that $E$ is isomorphic to the direct image of the line bundle.

Proof. $\quad r \mid d(S)$ is an immediate consequence of Lemma (2.6). We prove the last assertion under the hypothesis that $S$ is of type II and $E$ is of rank 2. In this case we notice that $A=\operatorname{Spec}\left(\oplus_{i=2}^{3} K^{\otimes i}\right)$. By Lemma (2.6), $H^{0}\left(A, \operatorname{Hom}\left(\pi^{*} E, \pi^{*} E\right)\right) \simeq \oplus_{i=0}^{3} H^{0}\left(S, \operatorname{Hom}\left(E, E \otimes K^{\otimes i}\right)\right)$ has dimension $\geq 2$. Hence we have $\operatorname{Hom}(E, E \otimes K) \neq 0$, $\operatorname{Hom}(E, E \otimes K)$ or $\operatorname{Hom}\left(E, E \otimes K^{\otimes 3}\right) \neq 0$ since $\operatorname{Hom}(E, E)=C$. It follows that $\operatorname{Hom}(E$, $\left.E \otimes K^{\otimes 2}\right) \neq 0$. Since $E$ is stable, $E \simeq E \otimes K^{\otimes 2}$. Hence by Takemoto [13], the Corollary follows. The proofs are same for other cases.

LEMMA (2.8). Using the notation of Theorem (2.1.3), let $\rho$ be a 
linear representation of degree $r$ of the fundamental group $H$ of the Abelian variety $A$. Let $L$ be the line bundle defined by the represen tation $\rho$. Then the pull-back $\bar{g}_{5}^{*} L$ is defined by the representation $\rho^{\prime}: H$ $\rightarrow G L(r, C) g \rightarrow \rho\left(g_{5} g g_{5}^{-1}\right)$ where $\bar{g}_{5}$ is the automorphism of $A$ induced by $g_{5}$.

Proof. The lemma is an immediate consequence of the following commulative diagram:

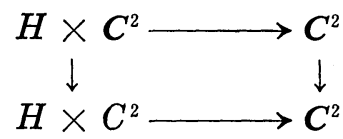

where horizontal arrows are the action of $H$ on the universal covering space $C^{2}$, the left vertical arrow sends $(g, x) \in H \times C^{2}$ to $\left(g_{5} g g_{5}^{-1}, g_{5} x\right)$ and the right vertical arrow is the action of $g_{5}$ on the universal covering space $C^{2}$ of the hyperelliptic surface $S$.

LEMMA (2.9). Using the notation of Theorem (2.1.3), let $L$ be a line bundle over a hyperelliptic surface $S$. Then $L$ is homologically equivalent to 0 if and only if it is numerically equivalent to 0.

Proof. If a line bundle is homologically equivalent to 0 , then generally it is numerically equivalent to 0 . Hence we assume that $L$ is numerically equivallent to 0 . We prove this for a hyperelliptic surface of type I i). In other cases, the proofs are similar. Let us consider the pull-back $\pi^{*} L$ over $A$. Over an abelian variety, the numerical equivalence coincides with the homological equivalence. Hence there exists a unitary representation $\rho$ of degree 1 of the fundamental group $G$ of $A$ such that $\pi^{*} L$ is associated with $\rho$. We put $\rho\left(g_{1}\right)=\alpha, \rho\left(g_{2}\right)=\beta, \rho\left(g_{3}\right)=\gamma, \rho\left(g_{4}\right)=$ $\delta^{6}$ where $\alpha, \beta, \gamma$ and $\delta$ are complex numbers with $|\alpha|=|\beta|=|\gamma|=|\delta|=1$. By Lemma (2.1.5) and Lemma (2.8), $\bar{g}_{5}^{*} \pi^{*} L$ is given by the unitary representation $\rho^{\prime}: \rho^{\prime}\left(g_{1}\right)=\alpha, \rho^{\prime}\left(g_{2}\right)=\beta^{-1}, \rho^{\prime}\left(g^{\varepsilon}\right)=\gamma, \rho^{\prime}\left(g_{4}\right)=\delta^{-1}$. Since $\pi^{*} L$ is isomorphic to $\bar{g}_{5}^{*} \pi^{*} L$, we conclude $\beta= \pm 1, \delta= \pm 1$. If we put $\rho^{\prime \prime}\left(g_{1}\right)$ $\rho^{\prime \prime}\left(g_{1}\right)=\alpha, \rho^{\prime \prime}\left(g_{2}\right)=\beta, \rho^{\prime \prime}\left(g_{3}\right)=\gamma, \rho^{\prime \prime}\left(g_{4}\right)=\delta, \rho^{\prime \prime}\left(g_{5}\right)=\varepsilon$ with $\varepsilon^{2}=\alpha$, then by Lemma (2.1.5) $\rho^{\prime \prime}$ defines a unitary representation of degree 1 of the fundamental group $G$ of $S$. Since there are two such $\varepsilon$ 's, there are two line bundles on $S$ defined by unitary representations such that there pull-backs over $A$ are isomorphic to $\varphi^{*} L$, Hence $L$ should be one of them and defined by a unitary representation. Hence $L$ is homologically equivalent to 0 . 
Let us determine all the irreducible unitary representation of the fundamental group $G$ of a given hyperelliptic surface $S$. Let $\rho$ be an irreducible unitary representation of degree $r$ of the fundamental group $G$ of $S$. Then by Proposition (1.2) and Corollary (2.7), $r$ devides $d(S)$. Hence it is sufficient to consider only such $r$. We put $r \cdot s=d(S)$. Since the subgroup $H$, the fundamental group of the abelian variety $A$, is commutative we can diagonalies the restriction of $\rho$ to $H$. Hence we may assume

$$
\rho\left(g_{i}\right)=\left(\begin{array}{ccccc}
\rho_{1}\left(g_{i}\right) & & & \\
& \rho_{2}\left(g_{i}\right) & & 0 \\
0 & & \ddots & \\
& & & \rho_{r}\left(g_{i}\right)
\end{array}\right)
$$

$1 \leq i \leq 4$. If $E$ denotes the vector bundle defined by the representation $\rho$, then $\pi^{*} E$ is the direct sum of the line bundle $L_{i}$ defined by the representation $\rho_{i}$ of the fundamental group of $A$. Since $\bar{g}_{5}^{*} \pi^{*} E$ is isomorphic to $\pi^{*} E$, by Lemma (2.8), we conclude

$$
\begin{aligned}
& \rho_{j}(g)=\rho_{1}\left(g_{5}^{-j+1} g g_{5}^{j-1}\right), \quad 1 \leq j \leq r \\
& \rho_{1}(g)=\rho_{1}\left(g_{5}^{-r} g g_{5}^{r}\right) .
\end{aligned}
$$

Hence we proved

Proposition (2.10). The irreducible unitary representation is normalized as follows

$$
\rho\left(g_{i}\right)=\left(\begin{array}{llll}
\rho_{1}\left(g_{i}\right) & & & \\
& \rho_{1}\left(g_{5}^{-1} g_{i} g_{5}\right) & & \\
& & \ddots & \\
& & & \rho_{1}\left(g_{5}^{-r+1} g_{i} g_{5}^{r+1}\right)
\end{array}\right)
$$

$1 \leq i \leq 4, s \cdot r=d(S)$ where $\rho_{1}$ is a representation of degree 1 of the fundamental group $H$ of the abelian variety.

THEOREM (2.11). The irreducible unitary representation of the fundamental group $G$ of $S$ are classified as follows. (We give the values of generators $g_{1}, g_{2}, g_{3}, g_{5}$ and $g_{5}$ of $G$. All the matrices below are unitary.)

Case I i). $\quad S$ is of type I i).

$r=1 . \quad \rho\left(g_{1}\right)=\alpha, \quad \rho\left(g_{2}\right)= \pm 1, \quad \rho\left(g_{3}\right)=\gamma, \quad \rho\left(g_{4}= \pm 1\right.$,

$$
\rho\left(g_{5}\right)=\varepsilon \quad \text { with }|\alpha|=|\gamma|=1 \text { and } \varepsilon^{2}=\alpha \text {. }
$$




$$
\begin{aligned}
& r=2 . \quad \rho\left(g_{1}\right)=\left(\begin{array}{cc}
\alpha & 0 \\
0 & \alpha
\end{array}\right), \quad \rho\left(g_{2}\right)=\left(\begin{array}{cc}
\beta & 0 \\
0 & \beta^{-1}
\end{array}\right), \quad \rho\left(g_{3}\right)=\left(\begin{array}{ll}
\gamma & 0 \\
0 & \gamma
\end{array}\right), \\
& \rho\left(g_{4}\right)=\left(\begin{array}{cc}
\delta & 0 \\
0 & \delta^{-1}
\end{array}\right), \quad \rho\left(g_{5}\right)=\left(\begin{array}{cc}
0 & \alpha \\
1 & 0
\end{array}\right) \\
& \text { with } \beta^{2} \neq 1 \text { or } \delta^{2} \neq 1 \text {. }
\end{aligned}
$$

There is no irreducible unitary representation of degree $>2$.

Case I ii). $\quad S$ is of type I ii).

$$
\begin{array}{ll}
r=1 . \quad & \rho\left(g_{1}\right)=\alpha, \quad \rho\left(g_{2}\right)=1, \quad \rho\left(g_{3}\right)=\gamma, \quad \rho\left(g_{4}\right)= \pm 1, \\
& \rho\left(g_{5}\right)=\varepsilon \quad \text { with }|\alpha|=|\gamma|=1 \text { and } \varepsilon^{2}=\alpha . \\
r=2 . \quad & \rho\left(g_{1}\right)=\left(\begin{array}{cc}
\alpha & 0 \\
0 & \alpha
\end{array}\right), \quad \rho\left(g_{2}\right)=\left(\begin{array}{cc}
\beta & 0 \\
0 & \beta^{-1}
\end{array}\right), \quad \rho\left(g_{3}\right)=\left(\begin{array}{cc}
\gamma & 0 \\
0 & \beta^{-1} \gamma
\end{array}\right), \\
\rho\left(g_{4}\right) & =\left(\begin{array}{cc}
\delta & 0 \\
0 & \delta^{-1}
\end{array}\right), \quad \rho\left(g_{5}\right)=\left(\begin{array}{cc}
0 & \alpha \\
\alpha & 0
\end{array}\right) \\
\text { with } \beta \neq 1 \text { or } \delta \neq \pm 1 . &
\end{array}
$$

There is no irreducible unitary representation of degree $>2$.

Case II i). $\quad S$ is of type II i).

$r=1$.

$$
\begin{aligned}
& \rho\left(g_{1}\right)=\alpha, \quad \rho\left(g_{2}\right)=\rho\left(g_{4}\right)=\beta, \quad \rho\left(g_{3}\right)=\gamma, \quad \rho\left(g_{5}\right)=\varepsilon, \\
& \text { with }|\alpha|=|\beta|=|\varepsilon|=1, \varepsilon^{3}=\alpha, \beta^{3}=1 . \\
& \rho\left(g_{1}\right)=\left(\begin{array}{lll}
\alpha & 0 & 0 \\
0 & \alpha & 0 \\
0 & 0 & \alpha
\end{array}\right), \quad \rho\left(g_{2}\right)=\left(\begin{array}{ccc}
\beta & 0 & 0 \\
0 & \beta^{-1} \delta^{-1} & 0 \\
0 & 0 & \delta
\end{array}\right), \\
& \rho\left(g_{3}\right)=\left(\begin{array}{lll}
\gamma & 0 & 0 \\
0 & \gamma & 0 \\
0 & 0 & \gamma
\end{array}\right),
\end{aligned}
$$$$
r=3
$$

There is no other irreducible representation.

Case II ii). $S$ is of II ii).

$$
\begin{array}{ll}
r=1 . & \rho\left(g_{1}\right)=\alpha, \quad \rho\left(g_{2}\right)=1, \quad \rho\left(g_{3}\right)=\gamma, \quad \rho\left(g_{4}\right)=1, \\
& \rho\left(g_{3}\right)=\varepsilon, \quad \text { with }|\alpha|=|\gamma|=1 \text { and } \varepsilon^{3}=\alpha . \\
r=3 . & \rho\left(g_{1}\right)=\left(\begin{array}{lll}
\alpha & 0 & 0 \\
0 & \alpha & 0 \\
0 & 0 & \alpha
\end{array}\right), \quad \rho\left(g_{2}\right)=\left(\begin{array}{ccc}
\beta & 0 & 0 \\
0 & \beta^{-1} \delta^{-1} & 0 \\
0 & 0 & \delta
\end{array}\right),
\end{array}
$$




$$
\begin{aligned}
& \rho\left(g_{3}\right)=\left(\begin{array}{ccc}
\gamma & 0 & 0 \\
0 & \gamma \beta^{-1} & 0 \\
0 & 0 & \gamma \delta
\end{array}\right), \quad \rho\left(g_{4}\right)=\left(\begin{array}{ccc}
\delta & 0 & 0 \\
0 & \beta & 0 \\
0 & 0 & \beta^{-1} \delta^{-1}
\end{array}\right), \\
& \rho\left(g_{5}\right)=\left(\begin{array}{lll}
0 & 0 & \alpha \\
1 & 0 & 0 \\
0 & 1 & 0
\end{array}\right) \quad \text { with } \beta \neq 1 \text { or } \delta \neq 1 .
\end{aligned}
$$

There is no other irreducible unitary representation.

Case III i). $S$ is of type III i).

$r=1$.

$$
\rho\left(g_{1}\right)=\alpha, \quad \rho\left(g_{2}\right)=\rho\left(g_{4}\right)= \pm 1, \quad \rho\left(g_{3}\right)=\gamma, \quad \rho\left(g_{5}\right)=\varepsilon,
$$$$
\text { with }|\alpha|=|\gamma|=|\varepsilon|=1 \text { and } \varepsilon^{4}=\alpha \text {. }
$$

$r=2$

$$
\begin{aligned}
& \rho\left(g_{1}\right)=\left(\begin{array}{ll}
\alpha & 0 \\
0 & \alpha
\end{array}\right), \quad \rho\left(g_{2}\right)=\left(\begin{array}{ll}
\beta & 0 \\
0 & \delta
\end{array}\right), \quad \rho\left(g_{3}\right)=\left(\begin{array}{ll}
\gamma & 0 \\
0 & \gamma
\end{array}\right), \\
& \rho\left(g_{4}\right)=\left(\begin{array}{ll}
\delta & 0 \\
0 & \beta
\end{array}\right), \quad \rho\left(g_{5}\right)=\left(\begin{array}{ll}
0 & \varepsilon \\
1 & 0
\end{array}\right) \\
& \text { with }|\alpha|=|\gamma|=1, \beta^{2}=\delta^{2}=1, \varepsilon^{2}=\alpha, \text { and } \beta \neq \delta .
\end{aligned}
$$

$r=4$

$$
\begin{aligned}
& \rho\left(g_{1}\right)=\left(\begin{array}{llll}
\alpha & 0 & 0 & 0 \\
0 & \alpha & 0 & 0 \\
0 & 0 & \alpha & 0 \\
0 & 0 & 0 & \alpha
\end{array}\right), \quad \rho\left(g_{2}\right)=\left(\begin{array}{cccc}
\beta & 0 & 0 & 0 \\
0 & \delta^{-1} & 0 & 0 \\
0 & 0 & \beta^{-1} & 0 \\
0 & 0 & 0 & \delta
\end{array}\right) \text {, } \\
& \rho\left(g_{3}\right)=\left(\begin{array}{cccc}
\gamma & 0 & 0 & 0 \\
0 & \gamma & 0 & 0 \\
0 & 0 & \gamma & 0 \\
0 & 0 & 0 & \gamma
\end{array}\right), \quad \rho\left(g_{4}\right)=\left(\begin{array}{cccc}
\delta & 0 & 0 & 0 \\
0 & \beta & 0 & 0 \\
0 & 0 & \delta^{-1} & 0 \\
0 & 0 & 0 & \beta^{-1}
\end{array}\right) \text {, } \\
& \rho\left(g_{5}\right)=\left(\begin{array}{llll}
0 & 0 & 0 & \alpha \\
1 & 0 & 0 & 0 \\
0 & 1 & 0 & 0 \\
0 & 0 & 1 & 0
\end{array}\right)
\end{aligned}
$$

with either $\beta \neq \delta$ or $\beta \neq \pm 1$ or $\delta \neq \pm 1$.

There is no other irreducible unitary representation.

Case III ii). $S$ is of type III ii).

$r=1$.

$$
\rho\left(g_{1}\right)=\alpha, \quad \rho\left(g_{2}\right)=\rho\left(g_{4}\right)=1, \quad \rho\left(g_{3}\right)=\gamma, \quad \rho\left(g_{5}\right)=\varepsilon
$$$$
\text { with }|\alpha|=|\gamma|=1 \text { and } \varepsilon^{4}=\alpha \text {. }
$$

$r=2$

$$
\rho\left(g_{1}\right)=\left(\begin{array}{ll}
\alpha & 0 \\
0 & \alpha
\end{array}\right), \quad \rho\left(g_{2}\right)=\rho\left(g_{4}\right)=-\left(\begin{array}{ll}
1 & 0 \\
0 & 1
\end{array}\right) \text {, }
$$




$$
\begin{array}{rlrl}
\rho\left(g_{3}\right) & =\left(\begin{array}{cc}
\gamma & 0 \\
0 & -\gamma
\end{array}\right), \quad \rho\left(g_{5}\right)=\left(\begin{array}{ll}
0 & \varepsilon \\
1 & 0
\end{array}\right) \\
\text { with }|\alpha|=|\gamma|=1, \varepsilon^{2}=\alpha . & \\
r=4 . & \rho\left(g_{1}\right)=\left(\begin{array}{llll}
\alpha & 0 & 0 & 0 \\
0 & \alpha & 0 & 0 \\
0 & 0 & \alpha & 0 \\
0 & 0 & 0 & \alpha
\end{array}\right), & \rho\left(g_{2}\right)=\left(\begin{array}{cccc}
\beta & 0 & 0 & 0 \\
0 & \delta^{-1} & 0 & 0 \\
0 & 0 & \beta^{-1} & 0 \\
0 & 0 & 0 & \delta
\end{array}\right), \\
\rho\left(g_{3}\right) & =\left(\begin{array}{cccc}
\gamma & 0 & 0 & 0 \\
0 & \gamma \delta^{-1} & 0 & 0 \\
0 & 0 & \gamma \beta^{-1} \delta^{-1} & 0 \\
0 & 0 & 0 & \gamma \beta^{-1}
\end{array}\right), & \\
\rho\left(g_{5}\right) & =\left(\begin{array}{cccc}
0 & 0 & 0 & \alpha \\
1 & 0 & 0 & 0 \\
0 & 1 & 0 & 0 \\
0 & 0 & 1 & 0
\end{array}\right)=\left(\begin{array}{cccc}
\delta & 0 & 0 & 0 \\
0 & \beta & 0 & 0 \\
0 & 0 & \delta^{-1} & 0 \\
0 & 0 & 0 & \beta^{-1}
\end{array}\right), \\
\text { with } & \text { either } \beta^{2} \neq 1 \text { or } \beta \neq \delta^{-1} \text { or } \delta \neq 1 .
\end{array}
$$

There is no other irreducible unitary representation.

Case IV. $\quad S$ is of type IV.

$$
\begin{aligned}
& r=1 . \quad \rho\left(g_{1}\right)=\alpha, \quad \rho\left(g_{2}\right)=\rho\left(g_{4}\right)=1, \quad \rho\left(g_{3}\right)=\gamma, \quad \rho\left(g_{5}\right)=\varepsilon \\
& \text { with }|\alpha|=|\gamma|=1 \text { and } \varepsilon^{6}=\alpha \text {. } \\
& r=2 . \quad \rho\left(g_{1}\right)=\left(\begin{array}{ll}
\alpha & 0 \\
0 & \alpha
\end{array}\right), \quad \rho\left(g_{2}\right)=\rho\left(g_{4}\right)=\left(\begin{array}{ll}
\beta & 0 \\
0 & \beta^{2}
\end{array}\right) \text {, } \\
& \rho\left(g_{3}\right)=\left(\begin{array}{ll}
\gamma & 0 \\
0 & \gamma
\end{array}\right), \quad \rho\left(g_{5}\right)=\left(\begin{array}{ll}
0 & \varepsilon \\
1 & 0
\end{array}\right) \\
& \text { with }|\alpha|=|\gamma|=1, \beta \neq 1, \beta^{3}=1 \text { and } \varepsilon^{3}=\alpha \text {. } \\
& r=3 . \quad \rho\left(g_{1}\right)=\left(\begin{array}{ccc}
\alpha & 0 & 0 \\
0 & \alpha & 0 \\
0 & 0 & \alpha
\end{array}\right), \quad \rho\left(g_{2}\right)=\left(\begin{array}{ccc}
\beta & 0 & 0 \\
0 & \beta \delta & 0 \\
0 & 0 & \delta
\end{array}\right) \text {, } \\
& \rho\left(g_{5}\right)=\left(\begin{array}{ccc}
\gamma & 0 & 0 \\
0 & \gamma & 0 \\
0 & 0 & \gamma
\end{array}\right), \quad \rho\left(g_{4}\right)=\left(\begin{array}{ccc}
\delta & 0 & 0 \\
0 & \beta^{-1} & 0 \\
0 & 0 & \beta^{-1} \delta^{-1}
\end{array}\right) \text {, } \\
& \rho\left(g_{5}\right)=\left(\begin{array}{lll}
0 & 0 & \varepsilon \\
1 & 0 & 0 \\
0 & 1 & 0
\end{array}\right) \\
& \text { with }|\alpha|=|\gamma|=1, \beta^{2}=\delta^{2}=1, \varepsilon^{2}=\alpha \text { and either } \beta \neq 1 \\
& \text { or } \delta \neq 1 \text {. }
\end{aligned}
$$




$$
\begin{gathered}
r=6 . \quad \rho\left(g_{1}\right)=\alpha I_{6}, \quad \rho\left(g_{2}\right)=\left[\begin{array}{ccccccc}
\beta & 0 & 0 & 0 & 0 & 0 \\
0 & \beta \delta & 0 & 0 & 0 & 0 \\
0 & 0 & \delta & 0 & 0 & 0 \\
0 & 0 & 0 & \beta^{-1} & 0 & 0 \\
0 & 0 & 0 & 0 & \beta^{-1} \delta^{-1} & 0 \\
0 & 0 & 0 & 0 & 0 & \delta^{-1}
\end{array}\right], \\
\rho\left(g_{3}\right)=\gamma I_{6}, \quad \rho\left(g_{4}\right)=\left[\begin{array}{cccccc}
\delta & 0 & 0 & 0 & 0 & 0 \\
0 & \beta^{-1} & 0 & 0 & 0 & 0 \\
0 & 0 & \beta^{-1} \delta^{-1} & 0 & 0 & 0 \\
0 & 0 & 0 & \delta^{-1} & 0 & 0 \\
0 & 0 & 0 & 0 & \beta & 0 \\
0 & 0 & 0 & 0 & 0 & \beta \delta
\end{array}\right), \\
\rho\left(g_{5}\right)=\left(\begin{array}{llllll}
0 & 0 & 0 & 0 & 0 & \alpha \\
1 & 0 & 0 & 0 & 0 & 0 \\
0 & 1 & 0 & 0 & 0 & 0 \\
0 & 0 & 1 & 0 & 0 & 0 \\
0 & 0 & 0 & 1 & 0 & 0
\end{array}\right] \\
\text { with }|\alpha|=|\beta|=|\gamma|=|\delta|=1, \text { except for following two cases } \\
\text { (a) } \beta=\delta, \beta^{3}=\delta^{3}=1
\end{gathered}
$$

There is no other irreducible unitary representation.

Proof. The theorem follows easily from Lemma (2.1.5), (2.1.6), (2.1.7), (2.1.8), (2.1.9), (2.1.10), (2.1.11), (2.8) and Proposition (2.10).

THEOREM (2.12). Using the notation of Theorem (2.1.3), let $E$ be an $H$-stable vector bundle with numerically trivial Chern classes over $S$. Then $E$ is defined an irreducible unitary representation of the fundamental group $G$ of the hyperelliptic surface $S$.

Proof. If $E$ is of rand 1, the Theorem is nothing but Lemma (2.9). We prove the theorem under the assumption that $S$ is of type III i) and $r=2$. Proofs are similar in other case (in fact easier if $r=d(S)$ ). By Lemma (2.6), $\pi^{*} E$ is the direct sum of line bundles which are algebraically equivalent to 0 , where $\pi: A \rightarrow S$ is the natural projection. We put $S^{\prime}=S /\left(\bar{g}_{5}^{2}\right)$. Then we have a commutative diagram:

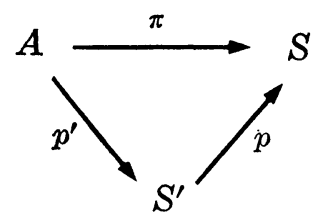


where $p$ and $p^{\prime}$ are the natural projections. We put $\pi^{*} E=L_{1} \oplus L_{2}$. Since $\bar{g}_{5}^{*} \pi^{*} E \simeq \pi^{*} E, \bar{g}_{5}^{*} L_{1} \oplus \bar{g}_{5}^{*} L_{2} \simeq L_{1} \oplus L_{2}$. It follows $\bar{g}_{5}^{*} L_{1} \simeq L_{1}$ or $\bar{g}_{5}^{*} L_{1}$ $\simeq L_{2}$. We shall show that we never have the first case $\bar{g}_{5}^{*} L_{1} \simeq L_{1}$ hence $\bar{g}_{5}^{*} L_{2} \simeq L_{2}$. If it were so, by the descent theory of Grothendieck there would be line bundles $L_{1}^{\prime} L_{2}^{\prime}$ over $S$ such that $\pi^{*} L_{1}^{\prime} \simeq L_{1}$ and $\pi^{*} L_{2}^{\prime} \simeq L_{2}$. Hence $\pi^{*} E \simeq \pi^{*}\left(L_{1}^{\prime} \oplus L_{2}^{\prime}\right)$. Taking $\pi_{*}, E \oplus E \otimes K \oplus E \otimes K^{\otimes 2} \oplus E \otimes K^{\otimes 3}$ $\simeq\left(L_{1}^{\prime} \oplus L_{2}^{\prime}\right) \oplus\left(L_{1}^{\prime} \oplus L_{2}^{\prime}\right) \otimes K \oplus\left(L_{1}^{\prime} \oplus L_{2}^{\prime}\right) \otimes K^{\otimes 2} \oplus\left(L_{1}^{\prime} \oplus L_{2}^{\prime}\right) \otimes K^{\otimes 3}$. Hence $E$ would be the direct sum of line bundles. This contradicts to the $H$ stability of $E$. We conclude $L_{2} \simeq \bar{g}_{5}^{*} L_{1}$ and $\bar{g}_{5}^{2 *} L_{1} \simeq L_{1}$ Hence $\pi^{*} E \simeq L_{1}$ $\oplus \bar{g}_{5}^{*} L_{1}$, with $\bar{g}_{5}^{*} L_{1} \neq L_{1}$ and $\bar{g}_{5}^{2 *} L_{1} \simeq L_{1}$. By Lemma (2.8), $\pi^{*} E$ is defined by the following unitary representation $\rho$ of the fundamental group $H$ of $A$ :

$$
\rho\left(g_{i}\right)=\left(\begin{array}{cc}
\rho_{1}\left(g_{i}\right) & 0 \\
0 & \rho_{1}\left(g_{5}^{-1} g_{i} g_{5}\right)
\end{array}\right), \quad 1 \leq i \leq 4,
$$

and $\rho_{1}\left(g_{5}^{-2} g_{i} g_{5}^{2}\right)=\rho_{1}\left(g_{i}\right)$ for any $1 \leq i \leq 4$ and $\rho_{1}\left(g_{5}^{-1} g_{i} g_{5}\right) \neq \rho_{1}\left(g_{i}\right)$ for some $i$. In view of Lemma (2.1.9), we conclude:

$$
\begin{array}{ll}
\rho\left(g_{1}\right)=\left(\begin{array}{ll}
\alpha & 0 \\
0 & \alpha
\end{array}\right), & \rho\left(g_{2}\right)=\left(\begin{array}{ll}
\beta & 0 \\
0 & \delta
\end{array}\right), \\
\rho\left(g_{3}\right)=\left(\begin{array}{ll}
\gamma & 0 \\
0 & \gamma
\end{array}\right), & \rho\left(g_{4}\right)=\left(\begin{array}{ll}
\delta & 0 \\
0 & \beta
\end{array}\right)
\end{array}
$$

with

$$
|\alpha|=|\gamma|=1, \beta^{2}=\delta^{2}=1 \text { and } \beta \neq \delta .
$$

If we put $\rho\left(g_{5}\right)=\left(\begin{array}{ll}0 & \varepsilon \\ 1 & 0\end{array}\right) \varepsilon^{2}=\alpha, \rho$ is extended to a unitary representation of the fundamental group $G$ of $S$. Since there are two $\varepsilon$ 's satisfying $\varepsilon^{2}=\alpha$, it follows there are two non-isomorphic vector bundle $E_{1}, E_{2}$ over $S$ such that $\pi^{*} E \simeq \pi^{*} E_{1} \simeq \pi^{*} E_{2}$. Hence $E$ should be one of them.

q.e.d.

COROLlary (2.13). Using the notation of Theorem (2.1.3), let $E$ be an $H$-stable vector bundle with numerically trivial Chern classes. Let $C_{n}$ be a generic hyperplane section of $n H$. Then the restriction $E_{n}$ of $E$ to $C_{n}$ is stable if $n$ is sufficiently big.

Proof. By Theorem (2.12), $E$ is defined by an irreducible unitary representation $\rho$ of $G$. By Lefschetz theorem, the canonical homomor- 
phism $i_{*}: \pi_{1}\left(C_{n}\right) \rightarrow \pi_{1}(S)=G$ is surjective if $n$ is sufficiently big (see Bott [2]). Hence the unitary representation $\rho \circ i_{*}$ is irreducible. Since $E_{n}$ is associated to this representation, by Seshadri [10], $E_{n}$ is stable.

CoRollary (2.14). Using the notation of Theorem (2.1.3), let $E$ be an $H$-stable vector bundle over $S$. Then the following are equivalent.

(1) $E$ is defined by an irreducible unitary representation of the fundamental group $G$.

(2) $E$ is defined by a unitary representation of the fundamental group $G$.

(3) $E$ is defined by a linear representation of the fundamental group $G$.

(4) $E$ has an integrable connection.

(5) $E$ has a connection.

(6) The Chern classes of $E$ are numerically trivial.

Proof. The implications (1) $\Rightarrow(2) \Rightarrow(3) \Rightarrow(4) \Rightarrow(5) \Rightarrow(6)$ follow from the general theory. (6) $\Rightarrow$ (1) follows from Theorem (2.12).

THEOREM (2.15). Using the notation of Theorem (2.1.3), let $F_{r}(S)$ be the set of isomorphism classes of $H$-stable vector bundles of rank $r$ with numerically trivial Chern classes over $S$. Then $F_{r}(S)$ has a complex analytic structure. More precisely,

Case I i). $S$ is of type I i).

If $r=1, \quad F_{r}(S) \simeq * \operatorname{Pic}^{0} S \amalg \mathrm{Pic}^{0} S \amalg \mathrm{Pic}^{0} S \amalg \mathrm{Pic}^{0} S$ $\simeq C /(1, \tau / 2) \amalg C /(1, \tau / 2) \amalg C /(1, \tau / 2) \amalg C /(1, \tau / 2)$.

If $r=2, \quad F_{r}(S) \cong C /(1, \tau) \times P^{1}(\omega), P^{1}(\omega)=P^{1}-\left\{Q_{1}, Q_{2}, Q_{3}, Q_{4}\right\}$, where $p: C /(1, \omega) \rightarrow \boldsymbol{P}^{1}$ is a double covering of $\boldsymbol{P}^{1}$ defined by a divisor of degree 2 over $C /(1, \omega)$ and $p$ is ramified at $Q_{1}, Q_{2}, Q_{3}$ and $Q_{4}$,

If $r \neq 1,2, \quad F_{r}(S)$ is empty.

Case I ii). $S$ is of type I ii).

If $r=1, \quad F_{r}(S) \simeq \operatorname{Pic}^{0} S \amalg \mathrm{Pic}^{0} S \simeq C /(1, \tau / 2) \amalg C /(1, \tau / 2)$.

If $r=2, \quad F_{r}(S)$ is an elliptic surface over $\Delta^{\prime}=\boldsymbol{P}^{1}-2$ points i.e. $F_{r}(S)$ is a non-compact complex manifold of dimension 2 with surjective map $p: F_{r}(S) \rightarrow \Delta^{\prime}$. Here $p$ is a proper morphism and $p^{-1}(x) \simeq C /(1, \tau)$ for a general point $x \in \Delta^{\prime}$.

If $r \neq 1,2, \quad F_{r}(S)$ is empty.

* $\amalg$ denotes disjoint union. 
Case II i). $S$ is of type II i).

If $r=1, \quad F_{r}(S) \simeq \operatorname{Pic}^{0} S \amalg \operatorname{Pic}^{0} S \amalg \operatorname{Pic}^{0} S$

$$
\simeq C /(1, \tau / 3) \amalg C /(1, \tau / 3) \amalg C /(1, \tau / 3) .
$$

If $r=3, \quad F_{r}=C /(1, \tau) \times \Delta^{\prime}$, where $\Delta^{\prime}$ is a non-singular and noncomplete curve.

If $r \neq 1,3, \quad F_{r}(S)$ is empty.

Case II ii). $S$ is of type II ii).

If $r=1, \quad F_{r}(S) \simeq \operatorname{Pic}^{0} S \simeq C /(1, \tau / 3)$.

If $r=3, \quad F_{r}(S)$ is an elliptic surface with the general fibre $C /(1, \tau)$ over a non-complete curve.

If $r \neq 1,3, \quad F_{r}(S)$ is empty.

Case III i). $S$ is of type III i).

If $r=1, \quad F_{r}(S) \simeq \operatorname{Pic}^{0} S \amalg \mathrm{Pic}^{0} S \simeq C /(1, \tau / 4) \amalg C /(1, \tau / 4)$.

If $r=2, \quad F_{r}(S) \simeq \mathrm{Pic}^{0} S^{\prime} \amalg \mathrm{Pic}^{0} S^{\prime} \simeq C /(1, \tau / 2) \amalg C /(1, \tau / 2)$,

where $S^{\prime} \simeq A /\left(\bar{g}_{5}^{2}\right)$.

If $r=4, \quad F_{r}(S) \simeq C /(1, \tau) \times \Delta^{\prime \prime}$, where $\Delta^{\prime \prime}$ is a non-singular and noncomplete curve.

If $r \neq 1,2,4, \quad F_{r}(S)$ is empty.

Case III ii). $S$ is of type III ii).

If $r=1, \quad F_{r}(S) \simeq \operatorname{Pic}^{0} S \simeq C /(1, \tau / 4)$.

If $r=2, \quad F_{r}(S) \simeq \operatorname{Pic}^{0} S^{\prime} \amalg C /(1, \tau / 2)$, where $S^{\prime} \simeq A /\left(\bar{g}_{5}^{2}\right)$.

If $r=4, \quad F_{r}(S)$ is an elliptic surface with the general fibre $C /(1, \tau)$ over a non-complete curve.

If $r \neq 1,2,3,4 \quad F_{r}(S)$ is empty.

Case IV. $S$ is of type IV.

If $r=1, \quad F_{r}(S) \simeq \operatorname{Pic}^{0} S \simeq C /(1, \tau / 6)$.

If $r=2, \quad F_{r}(S) \simeq \operatorname{Pic}^{0} S^{\prime} \amalg \mathrm{Pic}^{0} S^{\prime} \simeq C /(1, \tau / 3) \amalg C /(1, \tau / 3)$,

where $S^{\prime} \simeq A /\left(\bar{g}_{5}^{2}\right)$.

If $r=3, \quad F_{r}(S) \simeq \operatorname{Pic}^{0} S^{\prime \prime} \amalg \mathrm{Pic}^{0} S^{\prime \prime} \amalg \operatorname{Pic}^{0} S^{\prime \prime}$

$$
\simeq C /(1, \tau / 2) \amalg C(/ 1, \tau / 2) \amalg C /(1, \tau / 2),
$$

where $S^{\prime \prime} \simeq A /\left(\bar{g}_{5}^{3}\right)$.

If $r=6, \quad F_{r}(S)$ is an elliptic surface with the general fibre $C /(1, \tau)$ over a non-complete curve.

If $r \neq 1,2,3,6, \quad F_{r}(S)$ is empty. 
Proof. Case I i). If $r=1$, a line bundle $\in F_{r}(S)$ is defined by a unitary representation, $\rho\left(g_{1}\right)=\alpha, \rho\left(g_{2}\right)= \pm 1, \rho\left(g_{3}\right)=\gamma, \rho\left(g_{4}\right)= \pm 1, \rho\left(g_{5}\right)$ $=\varepsilon$ with $\varepsilon^{2}=\alpha$. This shows $F_{r}(S)=\operatorname{Pic}^{0} S \amalg \operatorname{Pic}^{0} S \amalg \mathrm{Pic}^{0} S \amalg \mathrm{Pic}^{0} S$ and $\mathrm{Pic}^{0} S \simeq C /(1, \tau / 2)$.

If $r=2$, Theorem (2.11) shows that such a bundle $E$ is defined by an irreducible unitary representation

$$
\begin{array}{ll}
\rho\left(g_{1}\right)=\left(\begin{array}{ll}
\alpha & 0 \\
0 & \alpha
\end{array}\right), & \rho\left(g_{2}\right)=\left(\begin{array}{cc}
\beta & 0 \\
0 & \beta^{-1}
\end{array}\right), \\
\rho\left(g_{3}\right)=\left(\begin{array}{ll}
\gamma & 0 \\
0 & \gamma
\end{array}\right), & \rho\left(g_{4}\right)=\left(\begin{array}{cc}
\delta & 0 \\
0 & \delta^{-1}
\end{array}\right), \\
\rho\left(g_{5}\right)=\left(\begin{array}{ll}
0 & \alpha \\
1 & 0
\end{array}\right) & \text { with } \beta^{2} \text { or } \delta^{2} \neq 1 .
\end{array}
$$

Remark that this normalization of irreducible unitary representation is not unique since representation

$$
\begin{array}{lll}
\rho\left(g_{1}\right)=\left(\begin{array}{ll}
\alpha & 0 \\
0 & \alpha
\end{array}\right), & \rho\left(g_{5}\right)=\left(\begin{array}{cc}
\beta^{-1} & 0 \\
0 & \beta
\end{array}\right), & \rho\left(g_{5}\right)=\left(\begin{array}{ll}
\gamma & 0 \\
0 & \gamma
\end{array}\right), \\
\rho\left(g_{5}\right)=\left(\begin{array}{cc}
\delta^{-1} & 0 \\
0 & \delta
\end{array}\right), & \rho\left(g_{5}\right)=\left(\begin{array}{ll}
0 & \alpha \\
1 & 0
\end{array}\right) &
\end{array}
$$

also defines $E$. These representations are equivalent. But this normalization shows that $E$ is the direct image of a line bundle $L$ of degree 0 over $A$. The condition $\beta^{2}$ or $\delta^{2} \neq 1$ shows that if $L \simeq p_{1}^{*} L_{1} \otimes p_{2}^{*} L_{2}, L_{2}^{\otimes 2}$ $\neq O_{C_{2}}$ where $p_{i}$ is the projection from $A=C /(1, \omega) \times C /(1, \tau)=C_{1} \times C_{2}$ onto the $i$-th factor. Let $W$ be the set of fixed points on $\hat{A}$ with respect to the action of $\bar{g}_{5}^{*}$. Then $F_{r}(S)=\hat{A}-W\left(\bar{g}_{5}^{*}\right)$. Since the action of $\bar{g}_{5}^{*}$ of $\hat{A}$ is given by

$$
\begin{aligned}
C_{1} \times C_{2} & \longrightarrow C_{1} \times C_{2} \\
(x, y) & \longmapsto(x,-y),
\end{aligned}
$$

the result follows.

By Theorem (2.11) and Theorem (2.12), or simply by Corollary (2.7), $F_{r}(S)$ is empty if $r \neq 1,2$.

Case I ii). If $r=1$, the proof is similar to the Case I i). Hence we omit it.

If $r=2$, the proof is similar to the Case I i) but delicate. Hence we give it. Let $\hat{A}$ be the dual abelian variety of $A$. Then $\bar{g}_{5}$ induces an automorphism $\bar{g}_{5}^{*}$ on $\hat{A}$. Let $W$ be the set of fixed points on $\hat{A}$ with 
respect to the action of the cyclic group $\left(\bar{g}_{5}^{*}\right)$. Then $F_{r}(S)=\hat{A}-W /\left(\bar{g}_{5}^{*}\right)$ by Theorem (2.11). We determine the variety $\hat{A} /\left(\bar{g}_{5}^{*}\right)$. To do this, we write down the abelian variety $\hat{A}$, the automorphism $\bar{g}_{5}^{*}$ and the subvariety $W$ explicitly. $\hat{A}$ is given by the period matrix $\left(\begin{array}{llll}1 & 0 & \tau & \frac{1}{2} \\ 0 & 1 & 0 & \omega\end{array}\right)$. To see this, let $B: C^{2} \times C^{2} \rightarrow C$ be an $R$-linear pairing $B\left(\left(x_{1}, x_{2},\left(y_{1}, y_{2}\right)\right)\right.$ $\left.=(1 / \operatorname{Im} \tau) x_{1} \bar{y}_{1}+(1 / \operatorname{Im} \omega) x_{2} \bar{y}_{2},\left(x_{1}, x_{2}\right), y_{1}, y_{2}\right) \in C^{2}$. Then it is easy to see that $B$ satisfies the following:

$1^{\circ} \quad B$ is complex linear on $\left(x_{1}, x_{2}\right)$ and complex anti-linear on $\left(y_{1}, y_{2}\right)$.

$2^{\circ} B$ is non-degenerate.

$3^{\circ}$ If we put $\operatorname{Im} B=\beta, \beta$ is integral on $U_{1} \times U_{2}$, where $U_{1}$ is the lattice defined by the column vectors of the period matrix $\left(\begin{array}{cccc}1 & 0 & \tau & 0 \\ 0 & 1 & \frac{1}{2} & \omega\end{array}\right)$ of $A$ and $U_{2}$ is the lattice defined by the column vectors of the period matrix $\left(\begin{array}{llll}1 & 0 & \tau & \frac{1}{2} \\ 0 & 1 & 0 & \omega\end{array}\right)$.

$4^{\circ} \quad U_{1}$ and $U_{2}$ are dual lattices under $\beta$.

Hence by Mumford p. 86 [7], $\hat{A}$ is given by the period matrix $\left(\begin{array}{llll}1 & 0 & \tau & \frac{1}{2} \\ 0 & 1 & 0 & \omega\end{array}\right)$. Let us now describe the automorphism $\bar{g}_{5}^{*}$. Since a translation $T_{x}, x \in A$ induces the identity on $\hat{A}$, we may assume $g_{5}$ is given by $g_{5}:(u, \zeta) \rightarrow$ $\left(x_{1},-x_{2}\right)$ using the notation above. By Mumford p. 85 [7], the line bundle corresponding to the point $\left(y_{1}, y_{2}\right) \in \hat{A}$ (or the universal covering space of $\hat{A})$ is given by the representation

$$
\begin{aligned}
& U_{1} \longrightarrow C \\
& w \\
&\left(u_{1}, u_{2}\right) \longmapsto \exp \left(-2 \pi i \beta\left(\left(u_{1}, u_{2}\right),\left(y_{1}, y_{2}\right)\right)\right) .
\end{aligned}
$$

Hence the automorphism $\left(x_{1}, x_{2}\right) \mapsto\left(x_{1},-x_{2}\right)$ of $A$ induces, the automorphism $\left(y_{1}, y_{2}\right) \mapsto\left(y_{1},-y_{2}\right)$ on $\hat{A}$. We regard the abelian variety $\hat{A} \simeq$ $C /\left\{(1,0),(0,1),(\tau, 0),\left(\frac{1}{2}, \omega\right)\right\}$ as an elliptic surface over an elliptic curve $\boldsymbol{C} /(1, \omega)$ by considering the projection $p_{2}: \boldsymbol{C}^{2} \rightarrow \boldsymbol{C}$. Then $\hat{A}$ has trivial functional and homological invariants. But $\hat{A}$ is not a basic elliptic surface. Let $\pi^{\prime}: \hat{A} \rightarrow C /(1, \omega)$ be this fibration, then the action of the cyclic group $\left(\bar{g}_{5}^{*}\right)$ preserves this fibration i.e. the diagram

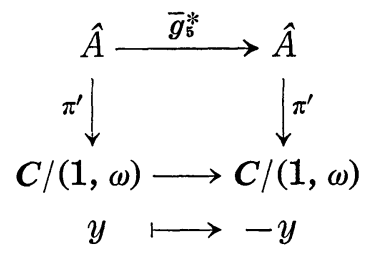


The fixed points should be contained in the fibres of [0], [1/2], [ $\omega / 2]$ and $[\omega / 2+1 / 2]$ where $[a]$ denotes the image of $a \in C$ into $C /(1, \omega)$. The points of the fibres over [0] and [1/2] are really fixed but the points over [ $\omega / 2]$ and $[\omega / 2+1 / 2]$ are not fixed. The action of $\bar{g}_{5}^{*}$ on $\pi^{-1}([\omega / 2])$ and $\pi^{-1}([\omega / 2+1 / 2])$ is the addition by [1/2] on the elliptic curve $C /(1, \tau)$. Hence $W=\pi^{-1}([0]) \cup \pi^{-1}([1 / 2])$. Let us consider the quotient space $\hat{A} /\left(\bar{g}_{5}^{*}\right)$. It has a fibration $\hat{A} /\left(\bar{g}_{5}^{*}\right) \rightarrow \boldsymbol{P}^{1}=$ the quotient space of the elliptic curve with respect to the action $y \mapsto-y$. Let $P_{1}, P_{2}, P_{3}$ and $P_{4}$ be images of

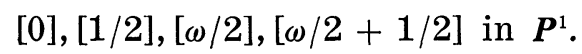

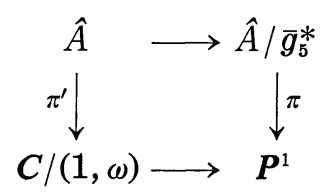

It is not difficult to see that the fibration $\pi$ is not singular at $P_{1}$ and $P_{2}$ and is singular at $P_{3}$ and $P_{4}$. The homological and functional invariants of $\hat{A} /\left(\bar{g}_{5}^{*}\right)$ is trivial. The general fibre $C /(1, \tau)$ is patched by the translation $T_{[1 / 2]}$ on $C /(1, \tau)$ when we consider small circles around $P_{3}$ and $P_{4}$. We proved $F_{r}(S)$ is an elliptic surface with general fibre $C /(1, \tau)$ over $P^{1}-2$ points and has two singular fibres.

Case II i). $S$ is of type II i).

If $r=1$, the proof is similar to the Case I i).

If $r=3$, the proof is similar to the Case $\mathrm{I}$ i).

Case II ii). $S$ is of type II ii).

If $r=1$, the proof is similar to the Case $I$ i).

If $r=2$, the proof is similar to the Case I ii) but much easier since the assertion in this case is weaker. Of course we can determine the surface explitly as in the Case I ii) but we do not use it in the sequel.

Crse III i). $S$ is of type III i).

If $r=1$, the proof is similar to the Case I i), $r=1$.

If $r=2$, the proof is similar to the Case I i), $r=2$ or $r=1$.

If $r=3$, the proof is similar to the Case I i), $r=2$.

Case III ii). $S$ is of type III ii).

If $r=1$, the proof is similar to the Case $\mathrm{I}$ i), $r=1$.

If $r=2$, the proof is similar to the Case I ii), $r=2$.

If $r=4$, the proof is similar to the Case I ii), $r=2$. 
Case IV. $S$ is of type IV.

If $r=1$, the proof is similar to the Case $I \mathrm{i}), r=1$.

If $r=2$, the proof is similar to the Case III i), $r=2$.

If $r=3$, the proof is similar to the Case III i), $r=2$.

If $r=6$, the proof is similar to the Case I i), $r=2$.

Hence we omit them.

THEOREM (2.16). $\quad F_{r}(S)$ is the coarse moduli space for $H$-stable vector bundles of rank $r$ with numerically trivial Chern classes over $S$. More pricisely, let $T$ be a variety and if we are given a vector bundle $E$ over $T \times S$ such that the restriction $E_{t}$ of $E$ on $t \times S$ is $H$-stable of rank $r$ with numerically trivial Chern classes for any (closed) point $t \in T$, then there exists a morphism $\varphi: T \rightarrow F_{r}(S)$ such that $E_{t}$ is isomorphic to the vector bundle corresponding to $\varphi(t) \in F_{r}(S)$.

Proof. Our proof depends on the following

LEMMA (2.17). Using the notation above, for any closed point $t \in T$, there exist an étale neighbourhood $U_{t}$ of $t$ and a line bundle $L$ over $U_{t} \times S^{\prime}$ such that $E_{t}$ is isomorphic to the restriction on $t \times S$ of the direct image of $L$, where $S^{\prime}$ is a covering space of degree $r$ of $S$ uniquely determined by $r$.

Proof. We prove the lemma under the assumption that $S$ is of type I i), $r=2$. The proofs in other cases are similar. Since the problem is local, we may assume $T$ affine. Let $K$ be the canonical bundle of $S$. We denote by $K$ the pull back $p_{1}^{*} K$ over $S \times T$. Then $A \times T=$ $\operatorname{Spec}\left(O_{A \times T} \oplus K\right)$. Let $F$ be a coherent sheaf on $A \times T$. Then $p_{2^{*}} F \simeq$ $\left.\widehat{H^{0}(A \times T, F}\right)$ since $T$ is affine. On the other hand, let $f: A \times T \rightarrow S \times T$ be the covering map. We have

$$
\begin{aligned}
& H^{0}\left(A \times T, f^{*} \text { End } E\right)=H^{0}\left(S \times T, f_{*} f^{*} \text { End } E\right) \\
&=\bigoplus_{i=0}^{1} H^{0}\left(S \times T, \operatorname{Hom}\left(E, E \otimes K^{\otimes i}\right)\right) .
\end{aligned}
$$

Since $\operatorname{dim}_{C} H^{0}\left(S, \operatorname{Hom}\left(E_{t^{\prime}}, E_{t^{\prime}} \otimes K^{\otimes i}\right)=1\right.$ for any point $t^{\prime} \in T, p_{2^{*}} \operatorname{Hom}(E$, $\left.E \otimes K^{\otimes i}\right)$ is locally free and of rank 1 . Hence if we take a sufficiently small open neighbourhood $U_{t}$ of $t, p_{2^{*}} \operatorname{Hom}\left(E, E \otimes K^{\otimes i}\right) \simeq O_{U_{t}}$. We may assume $T=U_{t}$. By the flat base change theorem,

$$
p_{2^{*}} \operatorname{Hom}\left(E, E \otimes K^{\otimes i}\right) \otimes k(t) \simeq \operatorname{Hom}\left(E_{t}, E_{t} \otimes K^{\otimes i}\right) \simeq C .
$$


Hence there exists an element $g$ of $\operatorname{Hom}(E, E \otimes K)$ such that $g$ induces an isomorphism on the fibre of $t$. Hence there exists an open neighbourhood $U_{t}$ of $t$ such that $g$ induces a non-zero homomorphism $g_{t^{\prime}} \in \operatorname{Hom}\left(E_{t^{\prime}}\right.$, $\left.E_{t^{\prime}} \otimes K\right)$ for any $t^{\prime} \in U_{t}$. Since $E_{t^{\prime}}$ and $E_{t^{\prime}} \otimes K$ are $H$-stable and have the same numerical Chern classes, $g_{t^{\prime}}$ is an isomorphism by Takemoto [12]. We may assume $T=U_{t}$. If we compose $g$ itself, we get $E \rightarrow E \otimes K \rightarrow E \otimes K^{\otimes 2}=E . \quad g^{2}$ is an isomorphism. Since $H^{0}(S \times T$, Hom $(E, E)) \stackrel{g^{2}}{\simeq} H^{0}\left(T, O_{T}\right), g^{2}$ is nothing but the multiplication by an element $f \in H^{\circ}\left(T, O_{T}^{*}\right)$. If we take an étale neighbourhood $U_{t}$ of $t$ such that $\sqrt{f}$ is a regular function, $H^{0}\left(A \times U_{t}\right.$, End $\left.t^{*} E\right) \simeq O_{U_{t}}[X] / X^{2}-1$. This shows $f^{*} E \simeq L_{1} \oplus L_{2}$. If we put $L=L_{1}$, then $L$ satisfies the condition.

q.e.d.

Proof of Theorem (2.16). We give the proof under the assumption that $\mathrm{S}$ is of type $\mathrm{I}$ i), $r=2$. By the Krull-Remack theorem and Lemma (2.6), the restriction $L_{A^{\prime}}$ of $L$ on the fibre $A \times t^{\prime}, t^{\prime} \in U_{t}$ is algebraically equivalent to 0 . Hence there exists a morphism $\psi_{U_{t}}: U_{t} \rightarrow \hat{A}$ such that $L_{A^{\prime}}$ is isomorphic to the line bundle corresponding to $\psi_{U^{\prime}}\left(t^{\prime}\right)$ for any $t^{\prime} \in U_{t} . \quad \psi_{U^{\prime}}$ does depend on the choice the étale neighbourhood $U_{t}$ and $L$. But the composition of the natural projection with $\psi_{U_{t}}: U_{t} \rightarrow \hat{A} \rightarrow$ $\hat{A} /\left(\bar{g}_{5}^{*}\right)$ is independent of the choice of $U_{t}$ and $L$. Hence we get a morphism $T \rightarrow F_{2}(S)$ satisfying the condition of the theorem. The proofs in other cases are similar hence we omit them.

THEOREM (2.18). If $r \geq 2$ and $r \mid d(S), F_{r}(S)$ gives local moduli for $S$ i.e. the $\operatorname{map} S \rightarrow F_{r}(S)$ locally separates the points of the local moduli space for $S$. If $S$ is neither of type I i) nor $\mathrm{I}$ ii), $F_{1}(S)$ gives local moduli for $S$.

Proof. Assume that $S$ is of type I i), $r=2$. By Suwa [11], the hyperelliptic surfaces of the type I i) are completely and effectively parametrized by $\tau$ and $\omega$. Let $S, S^{\prime}$ be hyperelliptic surfaces of type I i). Suppose that $F_{2}(S)$ and $F_{2}\left(S^{\prime}\right)$ are isomorphic. By Theorem (2.15), $F_{2}(S) \simeq C /(1, \tau) \times P^{1}-4$ points determined by $\omega, F_{2}\left(S^{\prime}\right) \simeq C /\left(1, \tau^{\prime}\right) \times P^{1}-4$ points determined by $\omega^{\prime}$. Let $\varphi: F_{2}(S) \rightarrow F_{2}\left(S^{\prime}\right)$ be an isomorphism. Let $x \in \boldsymbol{P}^{1}$ -4 points determined by $\omega$. Then the fibre $C /(1, \tau) \times x$ should be mapped onto a fibre by $\varphi$ since $\boldsymbol{P}^{1}-4$ points is not complete. Hence we get a 
morphism $\varphi^{\prime}: \boldsymbol{P}^{1}-4$ points determined by $\omega \rightarrow \boldsymbol{P}^{2}-4$ points determined by $\omega^{\prime}$ which makes the following diagram commutative,

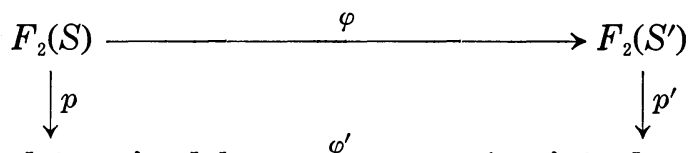

$\boldsymbol{P}-{ }^{1} 4$ points determined by $\omega \stackrel{\varphi^{\prime}}{\longrightarrow} \boldsymbol{P}_{1}-4$ points determined by $\omega^{\prime}$,

where $p, p^{\prime}$ are the projections. It follows that $C /(1, \tau) \simeq C /\left(1, \tau^{\prime}\right), C /(1, \omega)$ $\simeq C /\left(1, \omega^{\prime}\right)$, since the ramified covering $C /(1, \omega)$ (resp. $C /\left(1, \omega^{\prime}\right)$ ) is determined by 4 points where it ramifies. Hence $S \mapsto F_{2}(S)$ gives local moduli.

Assume now that $S$ is of type I ii), $r=2$. Let $S$ and $S^{\prime}$ be hyperelliptic surface of type I ii). By Theorem (2.15) $F_{2}(S)$ (resp. $F_{2}\left(S^{\prime}\right)$ ) is isomorphic to an elliptic surface over $\Delta$ (resp. $\Delta^{\prime}$ ) which is $\boldsymbol{P}^{1}-2$ points. The elliptic surface $F_{2}(S)$ has 2 singular fibres. Let $\varphi$ be an isomorphism of elliptic surfaces $F_{2}(S)$ and $F_{2}\left(S^{\prime}\right)$. Then as in the Case $I$ ii), $\varphi$ preserves the fibration hence it maps the singular fibres to the singular fibres. It follows that $\varphi$ induces an isomorphism of $\boldsymbol{P}^{1}-4$ points determined by $\omega$ and $P^{1}-4$ points determined by $\omega^{\prime}$. Therefore $C /(1, \tau) \simeq C /\left(1, \tau^{\prime}\right)$ and $C /(1, \omega) \simeq C /\left(1, \omega^{\prime}\right)$. Hence $F_{2}(S)$ gives local moduli for $S$.

The proof in other cases is similar and really is simpler since those surfaces are parametrized by one parameter $\tau$.

Remark (2.19). If $S$ is of type I i) or of I ii). $\quad F_{1}(S)$ does not give local moduli. $\quad F_{1}(S)$ is essentially the Picard scheme of $S$. Hence $F_{r}(S)$, $r \geq 2$ that can be regarded as a generalization the Picard scheme gives the finer result than the Picard scheme.

COROLlary (2.20). Any H-semi-stable vector bundle of rank 2 with numerically trivial Chern classes over $S$ which is not $H$-stable can not be a limit of H-stable vector bundles of rank 2 with numerically trivial Chern classes if $2 \neq d(S)$ (See Definition (2.21)). There exists for any hyperelliptic surface $S$ an $H$-semi-stable vector bundles of rank $r$ over $S$ which can not be a limit of $H$-stable vector bundles if $r \geq 2$.

Before we give proof we need

DEFINITION (2.21). Let $E$ be an $H$-semi-stable with numerically trivial Chern classes. We say that $E$ is a limit of $H$-stable vector bundles with numerically trivial Chern classes if there exist an irreducible variety 
$T$, a locally free sheaf $F$ over $S \times T$ and two points $t_{0}, t_{1} \in T$ such that $F_{t_{0}} \simeq E$ and $F_{t_{t}}$ is $H$-stable. We remark, then $F_{t}$ is $H$-stable on an open dense set $T$ since $H$-stability is an open condition (see Maruyama [4]).

LEMMA (2.22). Let $S$ denote a hyperelliptic surface as always. Let $E$ be an $H$-semi-stable vector bundle of rank 2 with numerically trivial Chern classes. Then the restriction $E_{1 C}$ of $E$ on any non-singular curve $C$ on $S$ is semi-stable.

Proof. Let $A$ be the abelian variety in Theorem (2.1.3). We use the notation of Definition (2.21). First of all we remark that $\pi^{*} E$ is homogeneous i.e. $\pi^{*} E \simeq T_{x}^{*} \pi^{*} E$ for any $x \in A$. In fact, if $E$ is $H$-stable, $\pi^{*} E$ is the direct sum of line bundles which are algebraically equivalent to 0 hence homogeneous. If $E$ is not $H$-stable but $H$-semi-stable, then $\pi^{*} E$ is not $H$-stable but $H$-semi-stable. It follows from Proposition (5.2) Takemoto [12] that $\pi^{*} E$ is homogeneous. Hence we conclude easily from Morimoto [6] that $\pi^{*} E_{\mid C^{\prime}}$ is semi-stable for any non-singular curve $C^{\prime}$ on $A$. If we consider the fibre product $\tilde{C}=C \times{ }_{s} A . \quad \tilde{C}$ is an unramified covering of degree $d(S)$ of $C$. Since $\pi^{*} E_{\mid C}$ is semi-stable, $E_{\mid C}$ is semistable.

q.e.d.

Proof of Corollary (2.20). Assume $r \nmid d(S)$, then there is no $H$-stable vector bundle of rank $r$ with numerically trivial Chern classes over $S$. No semi-stable vector bundle can be a limit of $H$-stable vector bundles of rank $r$ with numerically trivial Chern classes over $S$. There does exist an $H$-semi-stable vector bundle of rank $r$ with numerically trivial Chern classes over $S$, for example $\overbrace{O_{S} \oplus \cdots \oplus O_{S}}^{r}$. Now we assume $r \mid d(S)$ and $r \neq d(S)$. Consider the $H$-semi-stable $E=\overbrace{O_{S} \oplus \cdots \oplus O_{S}}^{r}$. We show that $E$ is not a limit of $H$-stable bundles of rank $r$. Assume that $E$ is a limit of $H$-stable vector bundles of rank $r$ with numerically trivial Chern classes, we shall show this leads to a contradiction. We use the same notation as in Definition (2.21). If we take a generic hyperplane section $C$ of $S$, then, by the Lefschetz theorem, we get a family of semi-stable vector bundles $F_{t \mid c}, t \in U$ over $C$ where, $U$ is an open subset of $T$ containing the point $t_{0}$, since the stability is an open condition. Moreover we assume, by Theorem (2.12), that if $F_{t}$ is $H$-stable, then $F_{t \mid C}$ is stable. Let $M(C, r)$ denote the moduli space of the semi-stables vector bundles 
of rank $r$ and of degree 0 over $C$. Then we get a morphism $\varphi: U \rightarrow$ $M(C, r)$ such that $F_{t} \simeq$ the vector bundle corresponding to $\varphi(t) \in M(C, r)$. $C$ is so determined that $F_{t}$ is $H$-stable if and only if $\varphi(t)$ is a stable point of $M(C, r)$. As in the proof of (2.16), we get a morphism $\psi: F_{r}(S)$ $\rightarrow M(C, r)$ such that the restriction to $C$ of the vector bundle corresponding to a point $u \in F_{r}(S)$ is isomorphic to the vector bundle corresponding to the point $\psi(u) \in M(C, r)$. We may assume $\psi(u)$ is stable for any $u \in F_{r}(S)$. Under our hypothesis $r \mid d(S), r \neq d(S), F_{r}(S)$ is complete by Theorem (2.15). Hence $\psi\left(F_{r}(S)\right)$ is a closed subset of $M(C, r)$. If we denote by $U^{S}$ the subset of the points $t$ of $U$ such that $F_{t}$ is $H$-stable, then $\varphi\left(U^{S}\right) \subset \psi\left(F_{r}(S)\right)$. Hence $\varphi(U) \subset \varphi\left(U^{S}\right) \subset \psi\left(F_{r}(S)\right)$. It follows that $E$ is $H$-stable. This is a contradiction. It should be remarked that we use only the hypothesis that the restriction $E_{1 C}$ is semi-stable.

Assume now $r=d(S)$. We must show that there exist an $H$-semistable vector bundle of rank $r$ with numerically trivial Chern classes which is not limit of $H$-stable vector bundles. We show that the vector bundle $E=\overbrace{O_{S} \oplus \cdots \oplus O_{S}}^{r}$ can not be a limit of $H$-stable vector bundles. Assume that $E$ were a limit of $H$-stable vector bundles parameterized by $T$ as in Definition (2.21). Take a generic hyperplane section $C$ as above. We would get a morphism $\varphi: T \rightarrow M(C, R)$. On the other hand, under our hypothesis an $H$-stable vector bundle $E^{\prime}$ of rank $r$, with trivial Chern classes is a direct image of a line bundle over $A$ which is algebraically equivalent to 0 . Hence we have a family of $H$-semi-stable vector bundle over $S$ parametrized by $\hat{A}$ and this parametrization contains all the $H$-stable vector bundles of rank $r$ with numerically trivial Chern classes (cf. proof of Theorem 2.15). We get a morphism $\psi: \hat{A} \rightarrow$ $M(C, r)$. It would follow as above, $\varphi(T) \subset \varphi\left(T^{S}\right) \subset \psi(\hat{A})$. Hence there would be a line bundle $L$ over $A$ such that $L$ is algebraically equivalent to 0 and such that $E_{\mid C}$ and the restriction to $C$ the direct image $\pi_{*} L$ over $S$ correspond to the same point of the moduli space $M(C, r)$. Hence $\pi_{*} L_{\mid C}$ would have a filtration $0=G_{0} \subset G_{1} \cdots \subset G_{r}=\pi_{*} L_{\mid C}$ where $G_{i}$ is a sub-vector bundle of rank $i$ of $G_{i+1}$ with $G_{i+1} / G_{i} \simeq O_{C}$. This is impossible.

Let $E$ be an $H$-semi-stable vector bundle of rank 2 with numerically trivial Chern classes. We assume that $E$ is not $H$-stable. By Lemma (2.22) the restriction $E_{\mid C}$ is semi-stable. By the remark above, $E$ is not a limit if $H$-stable vector bundles.

q.e.d. 


\section{§ 3. Final remarks}

In view of $\S 1$, it seems interesting to study the irreducible unitary representations of the fundamental group of an algebraic variety. But it very often happens that there is no irreducible unitary representation of degree $\geq 2$ of the fundamental group. Concerning this, we ask

Problem 1. Under what hypothesis is the condition that a vector bundle is defined by an irreducible unitary representation open?

We ask

Problem $1^{\prime}$. If $H^{i}(\tilde{V}, Q)=0$ for $i \geq 1$, then is the condition above open? Here $\tilde{V}$ is the universal covering space of the variety.

Over a variety $V$ such that the condition above is open, the theory or irreducible unitary representation of the fundamental group seems interesting if it is not empty (see Proposition (1.3)).

\section{REFERENCES}

[1] Atiyah, M. F., Complex analytic connections in fibre bundles, Trans. Amer. Math. Soc., 85 (1957), 181-207.

[ 2 ] Bott, R., On a theorem of Lefschetz, Mich. Math. J., 6 (1959), 211-216.

[ 3 ] Grothendieck, A., Technique de descente et théorème d'existence en géométrie algébrique. I, Séminaire Bourbaki, $12(1959 / 60), \mathrm{n}^{\circ} 190$.

[4] Maruyama, M., Stable vector bundles on an algebraic surface, Nagoya Math. J., 58 (1975), 25-68.

[5] Matsushita, Y., Fibrés holomorphes sur un tore complexe, Nagoya Math. J., 14 (1959), 1-24.

[6] Morimoto, A., Sur la classification des espaces fibrés vectoriels holomorphes sur un tore complexe admettant des connections holomorphes, Nagoya Math. J., 15 (1959), 83-154.

[ 7 ] Numford, D., Abelian varieties, Oxford University Press (1970), London.

[ 8 ] Narasimhan, M. S. and Seshadri, C. S., Stable and unitary vector bundles on a compact Riemann surface, Ann. of Math., 82 (1965), 540-567.

[ 9 ] Oda, T., Vector bundles on an elliptic curve, Nagoya Math. J., 43 (1971), 41-72.

[10] Seshadri, C. S., Moduli of $\pi$-vector bundles over an algebraic curve, C. I. M. E., "Questions on algebraic varieties", Varenna 1969, 141-260, Edizioni Cremonese, Roma 1970.

[11] Suwa, T., On hyperelliptic surfaces, J. of the Faculty of Sci. Univ. of Tokyo, 16 (1969-1970), 469-476.

[12] Takemoto, F., Stable vector bundles on algebraic surfaces, Nagoya Math. J., 47 (1972), 29-48.

[13] — , Stable vector bundles on algebraic surfaces II, Nagoya Math. J., 52 (1973), 173-195.

[14] Umemura, H., Fibrés vectoriels positifs sur une courbe elliptique, Bull. Soc. Math. France, 100 (1972), 431-433. 
[15] Some results in the theory of vector bundles, Nagoya Math. J., 52 (1973), $97-128$.

[16] -9, A theorem of Matsushima, Nagoya Math. J., 54 (1974), 123-134.

[17] Weil, A., Généralisation des fonctions abéliennes, J. Math. Pures Appl., (9), 17 (1938), 47-87.

[18] — Variétés kählériennes, Hermann (1958) Paris.

Nagoya University 